\title{
Und ewig grüßt der Maulwurf
}

\section{Ein Kommentar zu Martin Kronauers Plädoyer für ein "Recht auf Arbeit»}

Zusammenfassung: Der Beitrag führt die in der PROKLA 197 begonnene Debatte über das bedingungslose Grundeinkommen fort. In seiner Auseinandersetzung mit Martin Kronauers Plädoyer für ein Recht auf Arbeit betont Stephan Lessenich das kritische Potenzial, das gerade in der - dem bürgerlichen Leistungsethos widersprechenden Bedingungslosigkeit des Grundeinkommens liegt. Es ist die Bedingungslosigkeit des Anspruchs auf materielle Existenzsicherung, die als das eigentliche Skandalon der Grundeinkommensdebatte gelten kann: Rechte zu denken, ohne im selben Atemzug korrespondierende Pflichten mitzudenken.

Schlagwörter: Bedingungsloses Grundeinkommen, Recht auf Arbeit, Gesellschaftskritik, Kapitalismuskritik

\section{»The mole grubs eternally»}

\section{A commentary on Martin Kronauer's plea for a right to work}

Abstract: This article continues the debate on the unconditional basic income that began in PROKLA 197. Here, in argument with Martin Kronauer's demand of a right to work, Stephan Lessenich stresses the critical potential of an unconditional basic income - its affront to the bourgeois ethos of achievement. For Lessenich it is the unconditionality of the right to a secure material livelihood, or the concept of rights without corresponding obligations, which is the real scandal of the basic income debate.

Keywords: Basic income, right to work, critique of society, critique of capitalism

$M$ artin Kronauer gebührt doppelter Dank-mindestens. ${ }^{1}$ Einerseits ein ganz persönlicher, holt der geschätzte Kollege doch mit seinem Beitrag in Heft 197 der PROKLA eine von mir vor über

* Stephan Lessenich ist Mitglied des Wissenschaftlichen Beirats der PROKLA. einem Jahrzehnt im Auftrag der Friedrich-Ebert-Stiftung verfasste Expertise (vgl. Lessenich 2009) aus der Versenkung, die seither in den unendlichen

1 Für wertvolle Hinweise zu diesem Text möchte ich zudem Mirko Broll und Markus Wissen danken. 
Weiten des Internets verschwunden war, ohne in dem der Stiftung ideologisch und strategisch verbundenen Parteiapparat für irgendeine nennenswerte Reaktion gesorgt zu haben. ${ }^{2}$ Andererseits entzieht sich Kronauer dankenswerterweise - trotz seiner eindeutigen Positionierung im Paradigmenstreit der um die Grundeinkommensidee herum etablierten »intellektuellen Bürgerkriegsordnung " (Lessenich 2009: 8): Jener »destruktive[n] Lust an der feindseligen Beschuldigung ", am »konfrontativen, wechselseitig delegitimierenden und desavouierenden Schlagabtausch von Befürwortern und Gegnern« (ebd.: 5), die schon damals die wissenschaftlich-politische Grundeinkommensdebatte prägte und der nicht wenige Kontrahent*innen (die aber, genau besehen, ausschließlich männlichen Geschlechts sind) auch heute noch hemmungslosen Lauf lassen.

In vielerlei Hinsicht ist zudem Kronauers Analyse inhaltlich zuzustimmen, und dies selbst dort, wo sie mit meinem eigenen Beitrag ins - wie gesagt: stets freundlich-konstruktive Gericht geht. So ist es auf der einen Seite völlig überzeugend, die normativen Stärken der (linken) Grundeinkommenskonzepte zum kritischen Maßstab zu erheben, an dem auch ein Plädoyer für das Recht auf Arbeit zu messen wäre: Dieses müsse gleichbedeutend damit sein, "ein Recht auf Lebensunterhalt zu haben, ohne sich entwürdigenden Zumutungen unter-

2 Immerhin wurde sie aber für die digitale Öffentlichkeit konserviert: Unter https:// library.fes.de/pdf-files/wiso/06193.pdf ist sie nach wie vor abrufbar (Zugriff: 15.1.2020). werfen zu müssen.« (625) ${ }^{3}$ Auf der anderen Seite wird von Kronauer zu Recht moniert, dass die Arbeit, genauer die konkrete Form ihrer Vergesellschaftung, bei Grundeinkommensbefürw orter*innen in aller Regel »eine Art Blackbox« (ebd.) bleibe. Die Antwort auf die Krise der gesellschaftlichen Organisation der Erwerbsarbeit werde gerade nicht in eben dieser Organisation selbst gesucht, sondern in der von dieser scheinbar entkoppelten Sphäre monetärer Verteilung. Eine solche Unterbelichtung der Frage der Arbeit bzw. genauer der Gretchenfrage, wer die gesellschaftlich notwendige Arbeit leistet und zukünftig leisten soll - und unter welchen Bedingungen -, gilt beziehungsweise galt in der Tat auch für meinen damaligen, in moderierender Absicht verfassten Beitrag.

So weit, so gut. Liegt Martin Kronauer aber richtig, wenn er die Verteilung der Arbeit zum zentralen, ja letztlich einzigen Gütekriterium gesellschaftspolitischer Transformationsprojekte erklärt? Geht "gesellschaftliche Teilhabe«, der normative Dreh- und Angelpunkt seiner Argumentation, in der verallgemeinerten Teilhabe am System gesellschaftlicher Arbeit auf?

\section{Ist ohne Arbeit alles nichts?}

Die Frage so zu formulieren, heißt Zweifel an Kronauers Antwort, und also an einem überzeugten »Ja«, anzubringen. Denn »Arbeit« ist bei ihm letzten Endes Erwerbsarbeit - »diejenige Form der Arbeit, mit der Geld verdient werden

3 Alle Seitenzahlen ohne weitere Angaben beziehen sich auf Zitate aus Kronauer 2019. 
soll $\aleph^{4}$. Die »Umgestaltung der Erwerbsarbeitsverhältnisse in einer Weise, dass alle an ihnen partizipieren und mindestens einen kulturell angemessenen Lebensunterhalt verdienen können" (619), das ist der regulative Fluchtpunkt von Kronauers Teilhabeprogrammatik. Warum aber der strikte Fokus auf $॥$ Erwerbsarbeit «? Oder genauer, und erstens: Warum nennt er das Kind nicht beim Namen? ${ }^{5}$ Denn es geht ihm doch faktisch um Teilhabe an Lohnabhängigkeit, um die Unterwerfung der Teilhabesuchenden unter ein soziales Herrschaftsverhältnis zum Zweck des Zugangs zu individuellen Lebenschancen und gesellschaftlich wertgeschätzten Gütern. Und zweitens: Was ist eigentlich mit - je nach theoriepolitisch bevorzugter Semantik - »Reproduktionsarbeit «, »Sorgearbeit«, »Care«?

»Solange kapitalistische Ökonomie vorherrscht«, da gibt es, ja kann es schlechterdings keinen Widerspruch geben zu Kronauers Analyse, »wird ein erheblicher Teil gesellschaftlich notwendiger Arbeit in der Form der Erwerbsarbeit erbracht.« (627) Aber dann kommt es: »hre Bedeutung für die Allgemeinheit wird gerade in der Form des Entgelts ausgedrückt. Sie dokumentiert, dass die erbrachte Ar-

4 So die knappe Definition der Bundeszentrale für politische Bildung, vgl. http:// www.bpb.de/nachschlagen/lexika/pocketpolitik/16404/erwerbsarbeit (Zugriff: 15.1.2020).

5 Beziehungsweise nur am Rande: Während von »Erwerbsarbeit « im Text $39 \mathrm{Mal}$ die Rede ist, fällt der Begriff der »Lohnarbeit « sieben-, jener der »Lohnabhängigkeit« (einschließlich Wortstammvariationen) gar nur viermal. beitsleistung in die Arbeitsleistung der Gesellschaft insgesamt eingegangen ist und gesellschaftlich etwas gilt. Sie weist damit über die unentgeltlich erbrachte und allein im privaten Kreis anerkannte Tätigkeit hinaus.« (Ebd., Herv.: i.o.) Was will uns das sagen? Welche analytische Position wird hier gegenüber privat und unentgeltlich erbrachten, re-produktiven Arbeitstätigkeiten eingenommen? Werden sie hiermit aus der Gesamtarbeitsleistung der Gesellschaft heraus definiert? Wird die empirisch gegebene gesellschaftliche Nicht-Geltung der nicht-entgoltenen Arbeit hier soziologisch verdoppelt? Werden mit einer solchen Darstellung nicht die herrschenden, kapitalistischen Wertmaßstäbe schlicht übernommen und in einem Akt »kapitalistischen Realismus « (Fisher 2013) reproduziert?

Und es schließen sich weitere Fragen an: Ist die Form des Entgelts - der Arbeitslohn - tatsächlich Ausdruck der allgemeinen Bedeutung der Arbeitsleistung? Oder nicht vielmehr, oder doch mindestens so sehr, Ausdruck ihrer partikularen Bedeutung, als Dienst nämlich an der privaten Profitaneignung? Stünde die - auch monetäre Wertschätzung des arbeitsteiligen Beitrags zur gesellschaftlichen Reproduktion nicht eher im Mittelpunkt einer nicht-kapitalistischen Gemein(wohl) ökonomie? Warum soll die erwerbsförmige bzw. erwerbsbezogene Verausgabung von Arbeitskraft sozial hochwertiger oder jedenfalls bedeutungsvoller sein als »allein im privaten Kreis anerkannte Tätigkeiten«? Warum spielt für Kronauers Wertschätzung der Erwerbsarbeit deren sozial wie ökologisch häufig destruktive Qualität kei- 
ne oder aber nur eine untergeordnete Rolle - wohingegen "private « Arbeit in Haushalten, Vereinen und Initiativen nicht selten damit beschäftigt ist, die Scherben erwerbsgesellschaftlich produzierter Zerstörungen aufzusammeln und wo möglich zu kitten?

Kronauers Antwort ist gewissermaßen eine politisch-strategische: Weil nur in der Erwerbsarbeit - im Lohnarbeitsverhältnis - die Quelle von Gegenmacht, das Zeug zur Konfliktfähigkeit und also das Potenzial zu gesellschaftlicher Transformation liege. Nur Erwerbsbeteiligung gebe die »Macht, in die Verhältnisse einzugreifen und diese zu ändern" (623), nur von »innen" könne man "auf die Verhältnisse selbst einwirken« (ebd.), nur erwerbsarbeitende Menschen erlangen »die Autonomie von politischen Subjekten« (ebd., Herv.: i.O.). Politik, so scheint es, wird hier ganz von der Ökonomie her gedacht - und Sozialität ebenso, denn "gesellschaftlich gebraucht « (624, Herv.: i.O.) werden aus dieser Perspektive nur Lohnarbeiter*innen, es gibt keinen der Lohnabhängigkeit vorgängigen oder äußerlichen Sozialstatus des Dazugehörens qua Gebrauchtwerdens. »Teil der Allgemeinheit « wird man also nur, »indem man in die sozialen Abhängigkeitsverhältnisse der Erwerbsarbeit eingebunden ist « (627), und erst diese Integration in die Erwerbsarbeitsteilung eröffnet »die Möglichkeit, sich nicht nur individuell, sondern eben als Teil dieser Allgemeinheit mit anderen zur Wehr zu setzen, die gesellschaftlichen Abhängigkeitsverhältnisse selbst auf's Korn zu nehmen und zu ändern.« (Ebd.)

\section{Machtlose Gegenmacht}

Hm. Wie aber lässt sich dann erklären, dass es nicht nur im Wilden Westen (sprich den USA), sondern auch in der spätkorporatistischen Vollerwerbsgesellschaft Deutschland einen riesigen Niedriglohnsektor gibt (Reusch u.a. 2018)? Wenn allein die Erwerbsarbeit dafür sorgen kann, »dass prekäre Beschäftigungsverhältnisse überwunden und Marktabhängigkeit und Kapitalmacht zurückgedrängt werden « (627): Ist dann die Tatsache, dass dies im jüngsten, mehr als bloß konjunkturellen Beschäftigungsboom gerade nicht passiert ist, sondern genau das Gegenteil, für eine kritisch-soziologische Analyse und Argumentation bedeutungslos?

Martin Kronauer setzt also alles auf das Pferd der Erwerbsarbeit, auf deren Rücken soziale Teilhabe - und zwar nicht nur für die besten Renn- und Springpferde im Stall, sondern auch für die konkurrenzgesellschaftlich lahmeren Gäule - kollektiv erkämpft werden könne. Die emanzipatorischen Potenziale des Grundeinkommens hingegen sieht er äußerst skeptisch. Es lohnt, seine Ausführung dieses Zweifels hier noch einmal vollständig in Erinnerung zu rufen: "Die Befreiung vom individuellen Zwang zur Erwerbsarbeit soll bereits im Rahmen ökonomischer Verhältnisse angegangen werden, die nach wir vor kapitalistische sind, also auf der Grundlage des privaten Eigentums an Produktionsmitteln beruhen und die Erzielung von Profiten bezwecken. Darin inbegriffen ist die Entscheidungsmacht der Kapitalseite, Arbeitskräfte einzustellen oder zu entlassen. Selbst linke und marxistische Verfechterinnen 
und Verfechter eines bedingungslosen Grundeinkommens gehen nicht davon aus, dass es zu dessen Verwirklichung zuallererst einer revolutionären Beseitigung kapitalistischer Eigentums und Produktionsverhältnisse bedürfte. Aber sie verbinden mit ihm die Erwartung größerer Freiräume neben und innerhalb kapitalistischer Verhältnisse, die wiederum subversiv zu deren Transformation genutzt werden könnten der alte Maulwurf lässt grüßen, um ein Bonmot von Marx aufzugreifen.« (618)

Diese Kritik an den Blütenträumen großer Teile der GrundeinkommensCommunity ist meines Erachtens vollkommen zutreffend und berechtigt: Bei der Vorstellung einer institutionalisierten Befreiung von falscher Arbeit wird die Gewinnrechnung ohne den Wirt gemacht, ohne Berücksichtigung der real existierenden Herrschaftsverhältnisse, innerhalb derer ein solcher kollektivindividueller Akt der Emanzipation imaginiert wird. Aber, und ohne nun in einen billigen, gar hämischen Retourkutschenmodus verfallen zu wollen, wie er ansonsten Usus im vermeintlich so sozialprogressiven Milieu ist: Exakt dieselbe Kritik - genauer: Strukturkritik - lässt sich eben auch auf Kronauers eigene Hoffnung auf das subversivtransformative Potenzial eines Rechts auf Arbeit münzen.

Lassen wir seine kritische Beobachtung noch ein zweites Mal Revue passieren, diesmal in (stellvertretend) selbstreflexiver Absicht - und siehe da, sie liest sich genauso treffend ${ }^{6}$ : Die Befreiung vom individuellen Zwang zu prekärer

6 Die entsprechend veränderten bzw. angepassten Passagen sind kursiv gesetzt.
Erwerbsarbeit soll bereits im Rahmen ökonomischer Verhältnisse angegangen werden, die nach wir vor kapitalistische sind, also auf der Grundlage des privaten Eigentums an Produktionsmitteln beruhen und die Erzielung von Profiten bezwecken. Darin inbegriffen ist die Entscheidungsmacht der Kapitalseite, den Arbeitskrafteinsatzzu gestalten. Selbst linke und marxistische Verfechterinnen und Verfechter eines Rechts auf Arbeit gehen nicht davon aus, dass es zu dessen Verwirklichung zuallererst einer revolutionären Beseitigung kapitalistischer Eigentums und Produktionsverhältnisse bedürfte. Aber sie verbinden mit ihm die Erwartung größerer Freiräume innerhalb ${ }^{7}$ kapitalistischer Verhältnisse, die wiederum subversiv zu deren Transformation genutzt werden könnten ....." Da ist er also wieder, der alte Maulwurf - und lässt neuerlich grüßen, ${ }^{8}$ diesmal aus der Innenwelt kapitalistischer Arbeitsverhältnisse.

\section{Der alte Maulwurf ist müde}

Aber, ach!: Vielleicht ist ja der eine wie der andere Maulwurf - dem Kant grundsätzlich die leicht dümmlichenttäuschungsresistente Mentalität der »vergeblich, aber mit guter Zuversicht auf Schätze grabenden Vernunft«

7 Der Zusatz »neben und « aus dem Originalzitat ist hier gestrichen worden.

8 Kronauer setzt mit diesem beiläufigen Hinweis generationstypische MEW-Kenntnisse voraus. Marx sah im 18. Brumaire die Revolution an den Grundfesten der alten Ordnung nagen und Europa im zukünftigen Jubel über dieses fleißige Nachtwerk vereint: "Brav gewühlt, alter Maulwurf!« (Vgl. Marx 1852, MEW 8: 196). 
zuschrieb $^{9}$ - auf seine alten Tage müde geworden, gar erschöpft!?

Wir werden sogleich abschließend darauf zurückkommen. Zuvor sei nur kurz, und hoffentlich auf die freundlich-konstruktive Art Martin Kronauers, auf die Widersprüche verwiesen, in die sich die Forderung nach einem »Recht auf Arbeit « - unter gegebenen gesellschaftsstrukturellen Bedingungen - ihrerseits verstrickt. Folgt man nämlich Kronauers politökonomischer Kritik der Grundeinkommensidee, so stellt sich unweigerlich die Frage, worum es überhaupt geht beziehungsweise zuallererst gehen muss: Um die Verteilung der Arbeit? Oder um die Verteilung der Profite? Ist ersteres bei Kronauer nur eine Metapher für letzteres? Oder ist die Problematik der Profitabhängigkeit gesellschaftlicher Arbeitsorganisation im Kapitalismus letztlich dessen eigene »Blackbox«? Weitergefragt: Fordert das Recht auf Arbeit unter gegebenen, kapitalistischen Verhältnissen wirklich »die gesellschaftliche Debatte darüber heraus, welche Arbeiten gesellschaftlich notwendig sind und sein werden" (621)? Oder führt es unmittelbar zur Produktion von noch mehr (bestenfalls) gesellschaftlich überflüssigen bzw. (wahrscheinlicher) sozial korrumpierenden und ökologisch zerstörerischen Waren? Damit verbunden: Müsste man nicht über das erwerbsgesellschaftliche System der Bedürfnisse reden? Welche gesellschaftlichen Arbeiten sind denn

9 Keine Sorge, dieses beiläufige Bonmotdropping ist keineswegs tieferer Textkenntnis der Kritik der reinen Vernunft geschuldet, sondern einem luziden Beitrag zum »Philosophischen Bestiarium« auf DeutschlandfunkKultur entnommen (vgl. Tirnthal 2018). tatsächlich "notwendig", welche sind tatsächlich »für die Reproduktion des gesellschaftlichen Lebens unhintergehbar « (621)? Wer bestimmt darüber? Und wer sollte darüber bestimmen? Nur die Arbeitenden? Oder gar nur die Arbeitenden, die für die Reproduktion des gesellschaftlichen Lebens (»wirklich«) unhintergehbare Arbeit leisten?

Vielleicht wird damit deutlich, dass noch einiges ungeklärt ist an einem zu institutionalisierenden $»$ Recht auf Arbeit «. Ich habe an anderer Stelle in der PROKLA (Lessenich 2019) von der sozialdemokratischen Überidentifikation mit den unmittelbar vorgefundenen, gegebenen und überlieferten gesellschaftlichen Umständen gesprochen und dies mit einem Bonmot von Adam Przeworski (1985: 217) illustriert: »Die Arbeiterklasse hat einen langen Weg zurückgelegt von der Absicht, das Lohnverhältnis abzuschaffen, bis zum Anliegen, niemand davon auszuschließen.« (Przeworski 1985: 217, Übers.: S.L.) Die Sozialdemokratie des 20. Jahrhunderts wurde zur glühendsten Verfechterin des einst bekämpften Lohnarbeitsverhältnisses und der sozialen Inklusion via Lohnabhängigkeit. Auch bei Martin Kronauer geht es um die Vollinklusion aller Menschen in das Lohnarbeitsverhältnis (die »Erwerbsarbeit«), ${ }^{10}$ um von dort aus - ja, was eigentlich? Das Lohnarbeitsverhältnis abzuschaffen? Oder aber es möglichst lohnarbeitendenfreundlich zu gestalten?

Womit wir bei der Kronauer'schen Leitfrage wären: Der Frage, wofür zu kämpfen sich heute lohnt. Mit dem Ver-

10 Das Wort »Arbeit" wird in Kronauers Beitrag 194 Mal erwähnt. 
weis auf Anthony Atkinsons programmatischen Vorschläge einer "Beschäftigungsgarantie « und eines »Partizipationseinkommens « zielt Kronauer auf den Kampf für eine arbeits- und sozialpolitische Rekonstruktion der in die Krise geratenen Lohnarbeitsgesellschaft. »Weniger arbeiten und besser, Arbeit für alle! « (630): Mit diesem Schlachtruf schließt sein Beitrag. Aber vielleicht sollte man ja doch eher an deren Dekonstruktion arbeiten - und für eine andere Produktions- und Reproduktionsweise streiten?

Dies wiederum mag nun einigen allzu gewollt antireformistisch und wohlfeil salonrevolutionär erscheinen. Dabei geht es mir keineswegs darum, pragmatische Politikansätze zu desavouieren, die die Arbeits- und Lebensbedingungen vieler Menschen spürbar verbessern könnten. Und selbstverständlich gilt für alle linken Positionen, sei es nun das Grundeinkommen oder ein Recht auf Arbeit, dass sie grundsätzlich in dem Dilemma stehen, im Kapitalismus gegen den Kapitalismus operieren zu müssen. Die Frage ist doch vielmehr, inwiefern transformativ gedachte Programmatiken tatsächlich über die herrschenden Verhältnisse kapitalistischer Vergesellschaftung hinausweisen. Und da kann die Grundeinkommensidee gegenüber dem Recht auf Arbeit besonders in einem Belang punkten - nämlich mit der Forderung nach Bedingungslosigkeit.

Es ist die Bedingungslosigkeit des Anspruchs auf materielle Existenzsicherung, die als »das eigentliche Skandalon der Grundeinkommensdebatte« (Lessenich 2009: 18) gelten kann. Es ist die Durchkreuzung der auch bei Kronauer zentralen Reziprozitätserwartung, die Ablehnung einer »Pflicht zur
Gegenleistung als Voraussetzung für die Anerkennung als Vollbürger « (628), die bei den schärfsten Kritiker*innen des Grundeinkommens bisweilen nachgerade vegetative Abwehrreaktionen zu Tage fördert. Rechte zu denken, ohne im selben Atemzug korrespondierende Pflichten mitzudenken: Dass dies das bürgerliche Leistungsethos - und damit einen normativen Kern kapitalistischer Vergesellschaftung - angreift, sollte womöglich doch für die Grundeinkommensidee einnehmen.

\section{Postskriptum}

Die Sozialfigur des »Bürgerschrecks« ist leider viel zu selten geworden, ja eigentlich ausgestorben. Es gälte, sie zu revitalisieren oder für das 21. Jahrhundert neu zu erfinden. Unvergessen etwa, wie der damalige Ton Steine Scherben-Manager Nikel Pallat 1971 - am Beginn der nicht enden wollenden Krise der Lohnarbeitsgesellschaft - sich in der WDRTalkshow »Ende offen « anschickte, mit der aus der Innentasche seines Jacketts gezückten Handaxt wild auf den Tisch des Hauses einzuhacken, um seine Opposition zum Gegebenen, Bestehenden, Akzeptierten auszudrücken (vgl. Kringiel 2011). »Und deswegen mach ich jetzt hier mal diesen Tisch kaputt«: In all seiner hilflosen Symbolik berührt dieser expressiv-voluntaristische Axtakt heute, in Zeiten von »Hart aber fair«, auf ungewohnt eindringliche Weise. ${ }^{11}$

11 Wer die Szene noch nie gesehen hat, möge dies unbedingt auf Youtube tun (z.B. unter https://www.youtube.com/watch?v=1nqf pLC4yDE, Zugriff: 15.1.2020); zumindest ein unwillkürlicher Lacheffekt ist garantiert. 
Zugegeben: Auch Pallat scheiterte an der Tücke des Objekts; der Tisch ging, dank seiner unverwüstlichen Resopalplatte, keineswegs kaputt. Doch hatte die Aktion, so viel ist gewiss, so ganz und gar nichts Maulwürfiges an sich. Vielleicht sollte auch die gesellschaftspolitische Transformationsdebatte sich zumindest vorübergehend von der Hoffnung auf die Dynamik der Subversion verabschieden und stattdessen lieber mal wieder kräftig auf den Putz hauen. Die Zeiten, so scheint mir, sind danach.

\section{Literatur}

Fisher, Mark (2013): Kapitalistischer Realismus ohne Alternative? Eine Flugschrift. Hamburg.

Kringiel, Danny (2011): Amok auf der Mattscheibe. URL: https://www.spiegel.de/ geschichte/die-groessten-tv-ausrasteramok-auf-der-mattscheibe-a-947402.html, Zugriff: 16.1.2020.

Kronauer, Martin (2019): Konzepte der Teilhabe: Bedingungsloses Grundeinkommen oder Recht auf Arbeit?, In: PROKLA 197 49(4): 617-630. DOI: https://doi.org/10. 32387/prokla.v49i197.1847.

Lessenich, Stephan (2009): Das Grundeinkommen in der gesellschaftspolitischen Debatte. Expertise im Auftrag der Friedrich-EbertStiftung. WISO Diskurs. Bonn.

- (2019): Es rettet sie kein höh'res Wesen: Die Sozialdemokratie schafft sich ab. In: PROKLA 196 49(3): 449-453. DOI: https:// doi.org/10.32387/prokla.v49i196.1837.

Marx, Karl (1852): Der achtzehnte Brumaire des Louis Bonaparte. In: Marx Engels Werke (MEW), Bd. 8. Berlin 1960: 194-207.

Przeworski, Adam (1985): Capitalism and Social Democracy. Cambridge.

Reusch, Jürgen / Lenhardt, Uwe / Kuhn, Joseph / Moritz, Benjamin (2018): Transformation der Arbeitswelt: Daten, Schwerpunkt, Trends. In: Schröder, Lothar / Urban, Hans-Jürgen (Hg.): Gute Arbeit Ausgabe 2019. Frankfurt/M.: 281-349.

Tirnthal, Johanna (2018): Der Maulwurf-Hoffnung in finsteren Zeiten. URL: https:// www.deutschlandfunkkultur.de/das-phi losophische-bestiarium-der-maulwurfhoffnung-in.2162.de.html?dram:article_ id=425275, Zugriff: 16.1 .2020 . 